\title{
Diabetes mellitus Tipo 1 na Ausência de Neuropatia Autonômica não Altera a Taxa de Sudorese no Exercício
}

\author{
Type 1 Diabetes Mellitus in the Lack of Autonomic Neuropathy \\ Does not Alter the Sudoresis Rate in Exercise
}

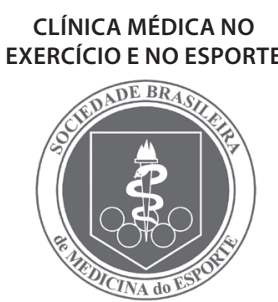

Artigo Original

\author{
Cristiane Martins Rocha ${ }^{1}$ \\ Luciana Gonçalves Madeira' \\ Kátia Regina Sá \\ Luciana Napoleão Lopes \\ Daniel Peixoto De Albuquerque ${ }^{1}$ \\ Leonardo Maurício Diniz² \\ Luiz Oswaldo Carneiro Rodrigues ${ }^{1}$
}

1. Laboratório de Fisiologia do

Exercício, Departamento de

Educação Física, Escola de Educação

Física Fisioterapia e Terapia

Ocupacional, Universidade Federal

de Minas Gerais, Belo Horizonte, MG

- Brasil.

2. Serviço de Endocrinologia e Metabologia do Hospital das Clínicas, Departamento de Clínica Médica, Faculdade de Medicina, Universidade Federal de Minas Gerais, Belo Horizonte, MG, Brasil.

\section{Endereço para correspondência: Luiz Oswaldo Carneiro Rodrigues, Universidade Federal de Minas \\ Gerais, Av. Carlos Luz, 4.664 - 31310-250 - Belo Horizonte, MG - Brasil. Tel./fax: 5531-3491-9460. E-mail: lor@ufmg.br}

Submetido em 13/02/2006 Versão final recebida em 08/07/2008 Aceito em 06/08/2008

\begin{abstract}
RESUMO
A sudorese é o principal mecanismo autonômico termorregulatório na espécie humana. A taxa de sudorese (TS) durante o exercício pode ser influenciada por diversos fatores, entre eles doenças, como o diabetes mellitus tipo 1, cujos pacientes são vítimas potenciais de distúrbios termorregulatórios. Os exercícios regulares são recomendados para diabéticos e, inclusive, alguns deles tornam-se atletas. No entanto, ainda não foi medida a TS de diabéticos durante exercício progressivo até a exaustão (GXT) e comparada com a de indivíduos sadios com características antropométricas semelhantes. O presente estudo comparou a TS de sete voluntários diabéticos (DM) e sete controles (GC), semelhantes quanto à idade, gênero, composição corporal e capacidade aeróbica. Antes e após o GXT, foram medidos a gravidade específica da urina (GEU), o peso e a glicemia capilar. A TS global foi calculada dividindo-se a variação do peso pelo tempo de coleta e área de superfície corporal. A TS local do antebraço foi calculada utilizando-se a variação do peso de um filtro absorvente corrigido pela sua área e tempo de coleta. As TS locais do antebraço e da perna foram induzidas por iontoforese com pilocarpina $(0,5 \%)$ no grupo DM. A freqüência cardíaca (FC) foi registrada antes, durante e após GXT. A temperatura média da pele (TMP) e as condições térmicas ambientais foram medidas durante o experimento. A glicemia capilar foi maior no DM, como o esperado. As TS global e local foram semelhantes entre os grupos, enquanto que a FC no repouso e a TMP no repouso e no exercício foram maiores no DM. Não houve diferença entre as TS do antebraço e da perna no DM com a pilocarpina. Concluiu-se que a resposta sudorípara foi semelhante entre diabéticos jovens e grupo controle durante exercício progressivo em ambiente temperado.
\end{abstract}

Palavras-chave: neuropatia autonômica diabética, termorregulação, glândula sudorípara, sudorese, exercício.

\begin{abstract}
Sudoresis is the main autonomic human thermoregulatory mechanism. It can be influenced by several factors, including diseases, among them diabetes mellitus Type 1 (DM1), whose patients are potential victims of thermoregulatory disturbances. Regular physical exercises are recommended to diabetic people and some of them even become athletes. However, up to now, the sweat rate (SR) of diabetic patients during graded exercise until exhaustion (GXT) has not been compared to the SR of healthy individuals with similar physical characteristics. The present study measured global (SRglobal) and local (SRlocal) sweat rates during GXT in 14 diabetic (DM) and control subjects (CG), similar in age, gender, body composition and aerobic capacity. Urine specific gravity (Gu), body mass and blood glucose (Gblood) were measured before and after GXT. Besides that, Srlocal (forearm and leg) was measured by iontophoresis with pilocarpine (0.5\%) in the DM group. Heart rate $(\mathrm{HR})$, skin temperature (Tsk) and environmental thermal conditions were measured during the experiment. Both initial and final Gblood were higher in the DM, as expected. The Gu were similar between groups. Exercise induced similar SRglobal and SRlocal in both groups, whereas the rest HR and rest and exercise Tsk were higher in the DM. Forearm and leg SR were not different in the DM. It was concluded that the sweating response was not different between young diabetic patients and control group during GXT in a temperate environment.
\end{abstract}

Keywords: diabetic autonomic neuropathy, thermoregulation, sweat glands.

\section{INTRODUÇÃO}

O calor produzido na atividade física pode elevar a temperatura corporal, o que aumenta a demanda dos mecanismos termorregulatórios para a transferência de calor do organismo para o ambiente. Em atividades de grande intensidade, a produção metabólica de calor pode ser 15 a 20 vezes maior do que a taxa metabólica basal, o que levaria a aumento de $1^{\circ} \mathrm{C}$ a cada cinco minutos, caso os mecanismos termorregulatórios não fossem ativados ${ }^{(1)}$. Em humanos, os principais mecanismos fisiológicos para aumentar a dissipação do calor nos ambientes quentes são a vasodilatação periférica e a produção e evaporação de suor, onde o calor é retirado da superfície corporal pela vaporização da água.
A taxa de sudorese (TS) depende do gasto energético, da duração e do tipo de atividade e das condições térmicas ambientais. A TS pode ser influenciada por fatores fisiológicos individuais como: idade ${ }^{(2)}$, gênero, hidratação, capacidade aeróbica, aclimatação( ${ }^{(3)}$ e pela presença de algumas doenças ${ }^{(4)}$, entre elas o diabetes mellitus tipo $1^{(5,6)}$.

Os exercícios físicos regulares são recomendados como parte do tratamento de indivíduos diabéticos ${ }^{(7)}$ e o mínimo recomendado para a melhora da saúde dessa população são 150 minutos por semana de atividade física moderada, entre 50 e 70\% da freqüência cardíaca máxima, distribuída regularmente em pelo menos três dias, com o ajuste apropriado no regime terapêutico ${ }^{(8)}$. Tal exercício pode representar aumento 
de três a dez vezes no metabolismo basal(9), o que significa produção moderada de calor a ser dissipado para o ambiente. Além disso, alguns pacientes com diabetes mellitus se tornam atletas e sua atividade física diária ultrapassa em duração e intensidade essa recomendação.

O diabetes mellitus tipo 1 é uma doença caracterizada pela destruição das células $\beta$ com deficiência da secreção de insulina, hiperglicemia crônica e complicações microvasculares, comprometendo o sistema nervoso periférico e autônomo. Seus pacientes são mais propensos a distúrbios termorregulatórios, como hipertermia e hipotermia, e a desidratação, o que acarreta maior risco esportivo para os atletas diabéticos que para os não diabéticos ${ }^{(5)}$. Além disso, a neuropatia autonômica diabética (NAD) é uma complicação comum e suas principais manifestações clínicas incluem taquicardia de repouso, hipotensão ortostática, constipação, disfunção erétil, intolerância ao exercício e disfunção sudorípara. Tais sintomas são resultado de uma disfunção no sistema nervoso autonômico, tanto parassimpático quanto simpáti$\mathrm{co}^{(10)}$. Atualmente, os testes utilizados para acessar a função autonômica são o teste quantitativo do reflexo do axônio sudomotor, possível de ser medido, mas tecnicamente complicado, ou a resposta simpática da pele, que não pode ser quantificada facilmente ${ }^{(11)}$. Portanto, seria de grande utilidade clínica um teste de triagem para acessar a presença da NAD e distúrbios termorregulatórios que seja de fácil manuseio e que possa ser realizado juntamente com outros testes já bem estabelecidos e amplamente utilizados nessa população, como o teste ergométrico.

Barany e Cooper ${ }^{(12)}$ demonstraram que, em indivíduos com DM, a estimulação por aquecimento passivo, injeções intradérmicas de acetilcolina ou corrente farádica resultavam em distribuição irregular da sudorese nos braços e pernas, inclusive com ausência de suor em algumas regiões. No entanto, a estimulação com calor direto sobre a pele resultou em sudorese normal, o que foi interpretado pelos autores como se as alterações na sudorese não fossem devidas a alterações na glândula sudorípara e sim à falha na estimulação ou condução nervosa.

Hoeldtke et al. ${ }^{(13)}$ realizaram um estudo longitudinal com diabéticos comparados com não diabéticos, para caracterizar a presença de disfunção sudorípara como um sinal precoce da disfunção neural periférica e da qualidade do controle habitual do nível glicêmico. Os autores observaram aumento relativo na TS do antebraço e diminuição nos membros inferiores, o que resultava em alteração importante na relação TS braços/pés dos diabéticos que apresentavam controle inadequado da glicemia. A conclusão dos autores foi que as respostas sudoríparas observadas foram indicativas da lesão autonômica. Além disso, sugeriram que o sistema nervoso autônomo simpático seria especialmente vulnerável aos efeitos adversos da hiperglicemia e que a disfunção simpática poderia ser precocemente detectada nos pacientes diabéticos tipo I através do teste da capacidade sudorípara com a estimulação farmacológica. No entanto, naquele estudo, o estímulo à sudorese representou tanto uma ativação indireta das glândulas pela acetilcolina, por sua ação nicotínica no axônio pós-ganglionar simpático, quanto uma ação direta nos receptores muscarínicos glandulares ${ }^{(14,15)}$. Portanto, a sudorese obtida não foi necessariamente somente um resultado da ativação simpática neural. Para que a TS seja indicativa da integridade do SNS como um todo, é indispensável uma estimulação central, através do exercício e/ou do aumento da temperatura corporal interna.

Um estudo anterior em nosso laboratório mostrou que a medida das taxas de sudorese global e no antebraço, durante o exercício progressivo até a exaustão (GXT), constitui indicador objetivo da capacidade sudorípara de um grupo de indivíduos normais como resultado da ativação do sistema nervoso simpático por estímulo térmico e não térmico (16). No entanto, até o momento, a taxa de sudorese de diabéticos não foi medida durante o GXT, para avaliar se ela poderia ou não ser utilizada como índice da integridade da função simpática periférica.

Portanto, pode-se presumir que o exercício progressivo até a exaustão é um estímulo central, térmico e não-térmico, e a iontoforese ou a injeção intradérmica de substâncias químicas são estímulos locais para a sudorese. O objetivo deste estudo foi comparar a resposta sudorípara, durante teste ergométrico máximo, entre indivíduos diabéticos jovens, sem confirmação de neuropatia autonômica periférica, e indivíduos normais com características físicas semelhantes, e, dessa forma, verificar se esse parâmetro pode ser um sinal precoce de triagem para a disfunção autonômica. Um segundo objetivo foi verificar a relação entre taxa de sudorese do antebraço/taxa de sudorese da perna durante estímulo com pilocarpina em pacientes diabéticos jovens.

\section{MÉTODOS}

Este estudo foi aprovado pelo Comitê de Ética em Pesquisa da UFMG e todos os voluntários assinaram o termo de consentimento livre e esclarecido. Participaram do experimento sete homens com diabetes mellitus tipo 1 (DM) e sete controles (GC) pareados nas variáveis idade, capacidade aeróbica e área de superfície corporal. Os critérios de exclusão eram a presença de neuropatia autonômica visceral grave, nefropatia diabética descompensada, doença cardíaca associada, hipertensão arterial ou outras complicações que pudessem comprometer a segurança dos voluntários durante a realização dos procedimentos ou causar possíveis alterações nos parâmetros avaliados nesta pesquisa. Além disso, outro critério de exclusão dos dados foi a presença de grandes variações no estresse térmico ambiental durante o teste, o que complicaria a comparação da resposta sudorípara.

Todos os diabéticos tinham tratamento médico regular individual. Foi realizado acompanhamento nutricional prévio aos testes, através de planejamentos dietéticos. Os indivíduos DM e GC foram considerados como naturalmente aclimatizados ao ambiente tropical quente e seco ${ }^{(3)}$. Foram medidas as variáveis antropométricas peso (Filizola ${ }^{\circledR}$ MF-100, e = 0,02kg, São Paulo, SP, Brasil), estatura, percentual de gordura $(\% \mathrm{G})^{(17)}$ e área de superfície corporal (ASC) ${ }^{(18)}$.

Os voluntários compareceram ao laboratório em dois dias, tendo como vestimenta short, meias e tênis. Eles foram orientados a ingerir $500 \mathrm{~mL}$ de água duas horas antes do experimento, para garantir o estado de hidratação no momento do teste ${ }^{(17)}$, e a evitar o consumo de tabaco, cafeína, bebidas alcoólicas e atividade física nas 24 horas anteriores. Os experimentos foram realizados no período da tarde, entre 12:00 e 14:30h. As temperaturas seca (Tseca) e úmida (Túmida) foram mantidas com o auxílio do ar condicionado (Cônsul ${ }^{\circledR}$ Air Master 7500 Btus, Manaus, AM, Brasil) e medidas de cinco em cinco minutos durante todo o tempo, desde a chegada do voluntário, em ambas as situações.

Foi escolhido como teste ergométrico a ser utilizado o GXT em cicloergômetro de frenagem mecânica Monark ${ }^{\circledast}$ (824 E, Varberg, Suécia) com carga inicial de 50 watts e aumentos de 25 watts a cada dois minutos, mantendo a 50rpm,até a exaustão ${ }^{(19)}$. A freqüência cardíaca (FC) foi monitorada através do cardiofrequencímetro (Polar ${ }^{\circledR}$ Vantage NV, Kempele, Finlândia) e registrada a cada dois minutos, desde o repouso, dois minutos antes de iniciar o exercício, até a recuperação, dois minutos depois de finalizado.

Os voluntários foram pesados antes e depois do teste em balança digital (Filizola ${ }^{\circledR}$ MF-100, e=0,02kg, São Paulo, SP, Brasil) e a taxa de sudorese global (TSG) foi calculada dividindo-se a diferença do peso pelo tempo entre as pesagens e depois relativizada pela ASC (TSG/ASC).

A produção de sudorese local foi medida utilizando uma câmara criada por plástico $\left(64 \mathrm{~cm}^{2}\right)$ fixado na região anterior do terço médio do antebraço direito (AD) utilizando-se esparadrapo, onde um papel-filtro absorvente $\left(16 \mathrm{~cm}^{2}\right.$ ) (J Prolab, SJ, dos Pinhais, PR, Brasil), anteriormente pesado, foi colocado através de uma abertura em uma das extremidades dessa câmara, que foi vedada em seguida ${ }^{(16)}$. Assim que o voluntário atingia a FC de 150bpm, o papel-filtro era substituído por outro, que seria retirado no período de recuperação do exercício. Esta FC foi escolhida para permitir a medida da sudorese durante a fase do exercício na vigência da ativação efetiva do sistema nervoso simpático ${ }^{(20)}$. Os filtros eram guardados em 
recipiente hermeticamente fechado e pesados em balança digital (Mettler ${ }^{\circledR}$ Toledo AB 204, escala analítica, e=0,001g, Columbus, OH, EUA) ao fim do experimento. A taxa de sudorese local (TSL) foi calculada dividindo a variação do peso pelo tempo de coleta e pela área de superfície do filtro.

A temperatura da pele foi medida em quatro regiões distintas do corpo: peito, braço, coxa e perna, através de termossensores de pele ligados ao teletermômetro (YSI, 400-A, Yellow Springs, OH, EUA). O registro foi feito a cada minuto desde o repouso até a recuperação. Para a determinação da temperatura média da pele (TMP) foi utilizada a equação proposta por Ramanathan(21).

A glicemia capilar foi medida no repouso, sendo considerada como a glicemia inicial $\left(G_{i}\right)$, e na recuperação, considerada a glicemia final $\left(G_{f}\right)$, utilizando glicosímetro (Accu-check Advantage Diagnostics Roche ${ }^{\oplus}$, Jaguaré, SP, Brasil). O controle glicêmico nas últimas semanas foi verificado utilizando a última dosagem da concentração de glicoemoglobina da rotina de acompanhamento clínico de cada voluntário, dosada em seu laboratório de análises de preferência. O estado de hidratação dos voluntários foi avaliado antes e após o exercício através da gravidade específica da urina (GEU), medida utilizando-se refratômetro (JSCP-Uridens, São Paulo, SP, Brasil).

Em uma segunda visita ao laboratório, o grupo DM teve a sudorese induzida por iontoforese (1,5mA, $60 \mu \mathrm{Acm}^{-2}$, durante cinco minutos) com $5 \mathrm{~mL}$ de solução de 0,5\% hidrocloridrato de pilocarpina (Galena Química e Farmacêutica, Campinas, SP, Brasil). A pilocarpina foi aplicada no terço médio da face anterior do antebraço direito (AD) e no terço proximal da face medial da perna direita (PD). O suor produzido foi medido por 15 minutos através do método descrito anteriormente para a coleta da TS local. Também foram medidas as temperaturas de pele em AD e PD e as condições térmicas ambientais.

\section{ESTATÍSTICA}

Os dados são apresentados como média e desvio padrão de sete indivíduos do grupo DM e sete do grupo CG. As variáveis TMP e FC foram analisadas utilizando-se ANOVA com medidas repetidas e Tukey como post-hoc. As demais variáveis foram analisadas utilizando-se o teste $t$ de Student. O nível de significância adotado foi de $p<0,05$.

\section{RESULTADOS}

Os indivíduos foram selecionados baseados em suas características físicas similares ( $p>0,05)$ : idade (24,0 $\pm 2,3$ e 24,4 $\pm 2,7$ anos), massa corporal (74,4 \pm 8,0 e 73,2 \pm 9,7kg), estatura (177,5 \pm 6,4 e 176,0 \pm 5,3cm), área de superfície corporal $\left(1,91 \pm 0,10\right.$ e 1,89 $\left.\pm 0,12 \mathrm{~cm}^{2}\right)$, gordura corporal (15,3 \pm 3,7 e 19,0 \pm 4,1\%) e capacidade aeróbica (33,4 \pm 5,2 e $\left.33,1 \pm 5,8 \mathrm{mLO}_{2} \cdot \mathrm{kg}^{-1} \cdot \mathrm{min}^{-1}\right)$, para os grupos DM e CG, respectivamente. O tempo de diagnóstico do grupo DM foi de $108 \pm 59$ meses.

As condições térmicas ambientais foram similares para os dois grupos ( $p>0,05)$ durante os experimentos: temperatura seca $(21,3 \pm 0,18 \mathrm{e}$ $\left.21,3 \pm 0,33^{\circ} \mathrm{C}\right)$; temperatura úmida $\left(16,9 \pm 0,22\right.$ e $\left.16,7 \pm 0,30^{\circ} \mathrm{C}\right)$ e Índice IBUTG $\left(18,1 \pm 0,28\right.$ e 17,7 $\left.\pm 0,77^{\circ} \mathrm{C}\right)$, para DM e GC, respectivamente.

Durante o teste ergométrico com exercício progressivo até a exaustão (GXT), a potência máxima atingida não foi diferente entre indivíduos diabéticos (189,3 \pm 31,8W) e indivíduos controle (192,9 \pm $27,8 \mathrm{~W}$ ). A freqüência cardíaca foi maior no grupo diabético durante o repouso $(p<0,05)$, mas foi similar à do controle durante o exercício e a recuperação $(p>0,05)$ (figura 1$)$.

A tabela 1 apresenta a TS global e local dos voluntários diabéticos e controles. Não houve diferença significativa entre os grupos pesquisados ( $p>0.05)$. Também não houve diferença entre as taxas de sudorese no antebraço e na perna $\left(2,15 \pm 1,24\right.$ e 1,40 $\pm 1,07 \mathrm{~g} \cdot \mathrm{m}^{-2} \cdot \mathrm{min}^{-1}$ respectivamente) dos pacientes com diabetes mellitus tipo 1.

A temperatura media da pele foi maior no grupo diabético durante o repouso e o exercício $(p<0,05)$, mas similar na recuperação $(p>0,05)$ (Figura 2). As temperaturas da pele do antebraço e da perna medidas durante a estimulação por iontoforese com pilocarpina não foram diferentes em indivíduos diabéticos (30,73 \pm 1,3 e 29,20 \pm 1,5, p > 0,05).

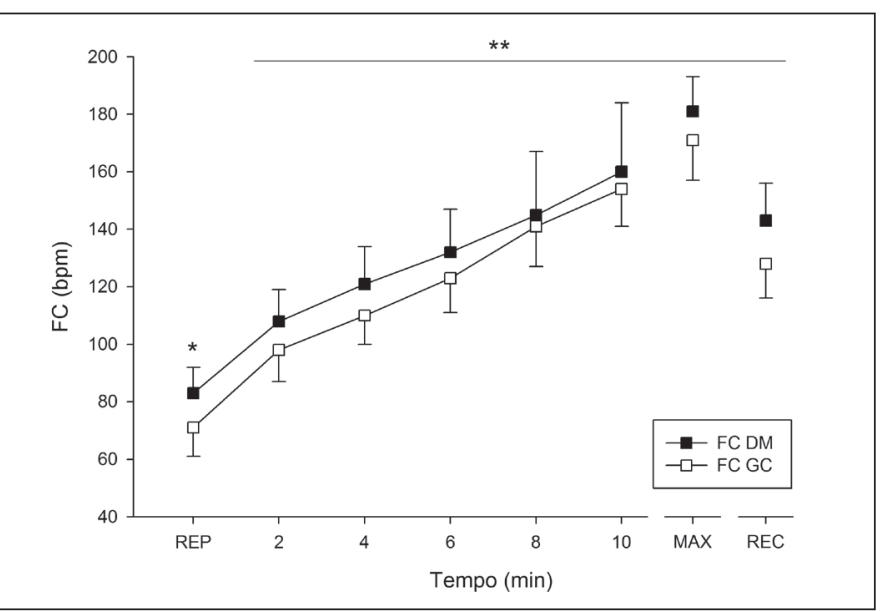

Figura 1. Frequência cardíaca (FC) durante repouso (REP), exercício e recuperação (REC). Dados em média \pm DP. * DM $>$ GC, $p<0,05 * *$ diferente REP, $p<0,05$.

Tabela 1. Taxa de sudorese global e local durante GXT em diabéticos e controles

\begin{tabular}{l|c|c|c|c}
\hline & \multicolumn{2}{|c|}{ Grupo controle $(\boldsymbol{n}=7)$} & \multicolumn{2}{c}{ Diabetes mellitus tipo $1(\boldsymbol{n}=7)$} \\
\hline TS global $\left(\mathrm{g} \cdot \mathrm{min}^{-1} \cdot \mathrm{m}^{-1}\right)$ & \multicolumn{2}{|c|}{$2,24 \pm 0,69$} & \multicolumn{2}{|c}{$2,55 \pm 0,42$} \\
\hline \multirow{2}{*}{ TS local $\left(\mathrm{g} \cdot \mathrm{min}^{-1} \cdot \mathrm{m}^{-1}\right)$} & $<150 \mathrm{bpm}$ & $>150 \mathrm{bpm}$ & $<150 \mathrm{bpm}$ & $>150 \mathrm{bpm}$ \\
\cline { 2 - 5 } & $0,60 \pm 0,58$ & $8,41 \pm 6,97$ & $0,44 \pm 0,30$ & $5,49 \pm 2,29$ \\
\hline
\end{tabular}

Dados apresentados em média \pm DP.

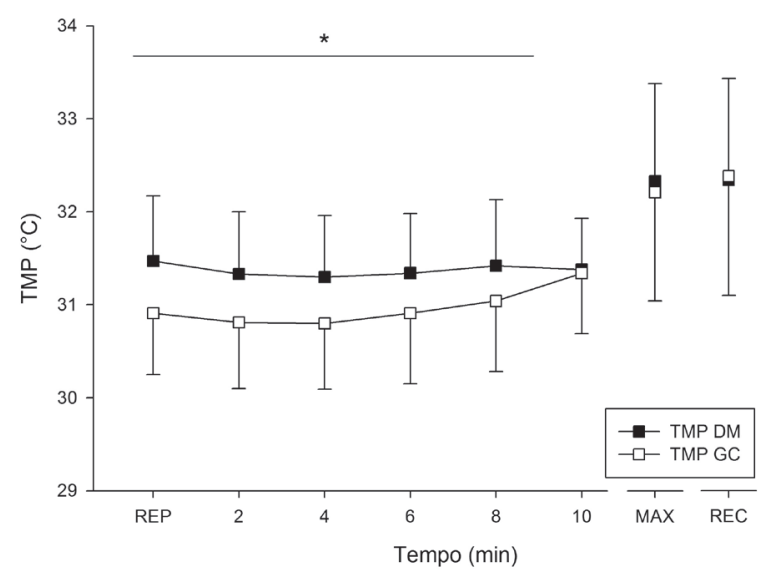

Figura 2. Temperatura média da pele (TMP) durante repouso (REP), exercício e recuperação (REC). Dados em média $\pm \mathrm{DP}$. * DM $>\mathrm{GC}, \mathrm{p}<0,05$.

Os níveis de glicoemoglobina no grupo diabético mostraram controle glicêmico inadequado nos últimos três meses (8,21 $\pm 1,95 \%)$, para valores de referência $<7,0 \%{ }^{(7)}$. A glicemia capilar inicial $(119,1 \pm 13,9)$ e a final $(122,1$ $\left.\pm 26,3 \mathrm{mg}_{\mathrm{dL}} \mathrm{L}^{-1}\right)$ foram maiores nos pacientes diabéticos que no grupo controle $\left(96,7 \pm 8,1\right.$ e $\left.88,3 \pm 10,6 \mathrm{mg}^{-\mathrm{dL}^{-1}}\right)(\mathrm{p}<0,05)$, como o esperado.

A gravidades específica das urinas inicial e final não foi diferentes entre os grupos diabético $(1,012 \pm 8,03$ e 1,014 $\pm 7,43)$ e controle $(1,011$ $\pm 9,78$ e 1,014 $\pm 7,31)$, respectivamente.

\section{DISCUSSÃO}

No presente estudo observou-se que não houve diferença entre diabéticos e normais na TS global e na TS local produzidas por estimulação central da glândula sudorípara alcançada com o exercício progressivo até a exaustão. Esse conjunto de dados sugere que não foi possível encontrar alterações sudoríparas em diabéticos jovens tipo 1 sem sinais de neuropatia autonômica periférica. Além disso, não houve diferença entre a TS no antebraço e na perna dos diabéticos, obtida com estimulação local direta da glândula sudorípara por pilocarpina, não sendo 
possível comprovar os efeitos da glicemia aumentada descritos por Hoeldtke et al. ${ }^{(13)}$ com a aplicação intradérmica de acetilcolina.

Ao contrário, o estudo realizado por Hoeldtke et al. ${ }^{(13)}$ encontrou respostas sudoríficas que poderiam significar um sinal precoce de neuropatia autonômica periférica no diabetes mellitus tipo 1. Naquele estudo, foram realizadas três avaliações, em um período de três anos, em diabéticos sem sinais de NAD (homens e mulheres) e em indivíduos normais como controles. Foi feita estimulação farmacológica da glândula sudorípara utilizando acetilcolina, com o indivíduo em repouso, em quatro locais do corpo: pé, região distal da perna, região proximal da perna e braço. Os resultados mostraram que a sudorese estava aumentada no braço dos diabéticos na primeira avaliação e que houve normalização com a melhora do controle glicêmico nas avaliações seguintes. Os autores afirmaram que os dados encontrados refletiam a integridade funcional do sistema nervoso autônomo simpático. No entanto, segundo Sato e Sato(22), a resposta simpática ocorre quando há uma estimulação central, através do exercício ou aumento da temperatura interna. Sendo assim, os resultados encontrados naquele estudo não estariam relacionados com o sistema nervoso simpático como um todo e sim com alterações periféricas, ou seja: no axônio pós- ganglionar da inervação simpática periglandular ou na própria glândula sudorípara. O presente estudo, ao estimular a TS utilizando um agente colinérgico com ação muscarínica direta na glândula sudorípara, não encontrou alterações na resposta funcional da glândula de diabéticos jovens. Por outro lado, a TS, tanto global quanto local, estimulada centralmente pelo sistema autonômico simpático, a partir de fatores térmicos e não-térmicos durante o exercício, também não foi diferente entre os grupos. Assim, não foi possível encontrar alterações sudomotoras centrais ou periféricas em diabéticos sem sinais de NAD, apesar do controle glicêmico também inadequado. A utilização de uma amostra mista de homens e mulheres pode ter influenciado as taxas de sudorese encontradas no estudo de Hoeldtke et al.(13), por haver diferença relatada na taxa de sudorese entre os $\operatorname{sexos}^{(23,24)}$, o que poderia contribuir para a contradição observada com o presente estudo.

A variação da temperatura média da pele indica que o grupo diabético respondeu com maior vasodilatação cutânea, o que já foi relatado previamente ${ }^{(25)}$. Por outro lado, Rendell et al. ${ }^{(26)}$ encontraram fluxo sanguíneo para pele diminuído em pacientes diabéticos medido com laser-doppler mas, diferentemente do estudo atual, seus achados foram em resposta a ambientes quentes $\left(35\right.$ e $40^{\circ} \mathrm{C}$ ). No entanto, no presente estudo, na recuperação, a temperatura da pele foi semelhante entre normais e diabéticos. Esse dado sugere que a estimulação central au- tonômica em resposta ao exercício e possível aumento da temperatura interna, semelhante para os dois grupos, teria superado esse efeito. No entanto, ainda não temos explicações para este achado.

A freqüência cardíaca foi maior no repouso nos diabéticos, mas semelhante no decorrer do exercício e na recuperação. A taquicardia de repouso, apresentada pelos voluntários diabéticos no presente estudo, constitui um único sinal clínico de neuropatia autonômica periférica, hipótese que, na ausência de outros indicadores, fica enfraquecida ${ }^{(7,10)}$.

Os dados do presente estudo indicam que não houve diferenças significativas, nas variáveis termorregulatórias estudadas, entre DM e GC, durante exercício progressivo máximo. Contudo, a ausência de tais diferenças associada ao tamanho da amostra utilizada recomenda novos estudos antes que conceitos definitivos sobre as respostas termorregulatórias do diabetes ao exercício progressivo possam ser estabelecidas. Outra limitação deste estudo foi a dificuldade de padronização da medida da hemoglobina glicada, o que pode ter interferido na definição clínica do grupo diabético. De qualquer forma, a grande freqüência com que os testes tinham que ser adiados porque os voluntários apresentavam hiper ou hipoglicemia na chegada ao laboratório (dos 19 dias marcados, foram sete coletas, sete adiamentos por hiperglicemia e cinco por hipoglicemia) indica que eram, de fato, pacientes malcontrolados. Finalmente, não foi possível garantir se a medida da TS durante um teste ergométrico seria um teste precoce de triagem da disfunção neural autonômica, uma vez que o presente estudo não comparou dois grupos diabéticos: um com e outro sem a disfunção autonômica. Por outro lado, se de fato o grupo estudado não é portador de disfunção autonômica, neste caso o teste foi capaz de medir a TS de forma adequada e foi fidedigno. Para aprofundarmos essas questões, é necessário um novo estudo no qual a amostra de diabéticos tenha a presença de NAD já diagnosticada.

Concluiu-se que um grupo de diabéticos jovens, sem sinais de neuropatia autonômica periférica, mesmo com controle glicêmico inadequado, apresentou resposta sudorípara semelhante à dos indivíduos não diabéticos, durante exercício progressivo até a exaustão em ambiente temperado.

\section{AGRADECIMENTOS}

Ao Conselho Nacional de Desenvolvimento Científico e Tecnológico (CNPq) e à Coordenação de Aperfeiçoamento de Pessoal do Nível Superior (Capes).

Todos os autores declararam não haver qualquer potencial conflito de interesses referente a este artigo.

\section{REFERÊNCIAS BIBLIOGRÁFICAS}

1. González-Alonso J, Teller C, Andersen SL, Jensen FB, Hyldig T, Nielsen B. Influence of body temperature on the development of fatigue during prolonged exercise in the heat. J Appl Physiol. 1999;86(3):1032-9.

2. Kenney WL. Thermorregulation at rest and during exercise in health older adults. Exerc Sport Sci Rev. 1997;25:41-76. Review

3. Aoyagi Y, McLellan TM, Shephard RJ. Interactions of physical training and heat acclimation. The thermophysiology of exercising in a hot climate. Sports Med. 1997;23(3):173-210. Review.

4. Pandolf KB, Sawka MN, Gonzalez RR, editors. Human performance physiology and environmental medicine at terrestrial extremes. 1st ed. Carmel (IN): Cooper Publishing Groups, 1986.

5. Chisholm DJ. Medical Problems in sport - Diabetes Mellitus. In: Sherry E, Wilson SF, editors. Oxford handbook of sports medicine. 1st ed. Oxford (NY): Oxford University Press, 1998.

6. Boulton AJ, Vinik Al, Arezzo JC, Bril V, Feldman EL, Freeman R, et al. Diabetic neuropathies: a statement by the American Diabetes Association. Diabetes Care. 2005;28(4):956-62. Review.

7. American Diabetes Association (ADA). Position Statements - Standards of Medical Care for Patiens with diabetes Mellitus. Diabetes Care. 2005;28:S04-S36.

8. American Diabetes Association (ADA). Standarts of Medical Care in Diabetes. Diabetes Care. 2006;29: S04-S42.

9. Ainsworth BE, Haskell WL, Leon AS, Jacobs Jr DR, Montoye HJ, Sallis JF, et al. Compendium of physical activities: classification of energy costs of human physical activities. Med Sci Sports Exerc. 2000;32:498-516.

10. Vinik Al, Maser RE, Mitchell BD, Freeman R. Diabetic autonomic neuropathy. Diabetes Care. 2003;26(5):1553-79. Review.

11. Hilz MJ, Hecht MJ, Berghoff M, Singer W, Neundoerfer B. Abnormal vasoreaction to arousal stimul - An early sign of Diabetic Sympathetic Neuropathy demonstrated by Laser Doppler Flowmetry. J Clin Neurophysiol. 2000;17:419-25.

12. Barany FR, Cooper EH. Pilomotor and sudomotor innervation in diabetes. Clin Sci (Lond). 1956;15(4):532-40.

13. Hoeldtke RD, Bryner KD, Horvath GG, Phares RW, Broy LF, Hobbs GR. Redistribution of sudomotor responses is an early sign of sympathetic dysfunction in type 1 diabetes. Diabetes. 2001;50(2):436-43.
14. Schmelz M, Schmidt R, Bickel A, Torebjork HE, Handwerker HO. Innervation territories of single sympathetic C fibers in human skin. J Neurophysiol. 1998;79(4):1653-60

15. Coon JM, Rothman S. The sweat response to drugs with nicotine like action. J Pharmacol Exp Ther 1941;73:1-11

16. Vimieiro-Gomes AC, Magalhães FC, Amorin FT, Machado-Moreira CA, Rosa MS, Lima NR, et al. Comparison of sweat rate during graded exercise and the local rate induced by pilocarpine. Braz J Med Biol Res. 2005;38(7):1133-9.

17. Brozek J, Grande F, Anderson T, Keys A. Densitometric analysis of body composition: revision of some quantitative assumptions. Ann N Y Acad Sci. 1963;110:113-40.

18. Dubois $D$, Dubois $E$. A formula to estimate the approximate surface area if height and weight be known. Arch Intern Med. 1916;17:863-71.

19. American College of Sports Medicine (ACSM). Position Stand - The prevention of thermal injuries during distance running. Med Sci Sports Exerc. 1995;19:529-33.

20. Rowell LB, O'Leary DS. Reflex control of the circulation during exercise: chemoreflexes and mechanoreflexes. J Appl Physiol. 1990,69(2):407-18. Review.

21. Ramanathan NL. A new weighting system for mean surface temperature of the human body. J Appl Physiol. 1964;19:531-3.

22. Sato K, Sato F. Individual variations in structure and function of eccrine sweat glands. Am J Physiol. 1983;32:971-81.

23. Shapiro Y, Pandolf KB, Avellini BA, Pimental NA, Goldman RF. Physiological responses of men and women to humid and dry heat. J Appl Physiol. 1980;49(1):1-8.

24. Anderson GS, Ward R, Mekjavik IB. Gender differences in physiological reactions to thermal stress. Eur J Appl Physiol Occup Physiol. 1995;71(2-3):95-101.

25. Bar-Or O, Rowland TW. Pediatric exercise medicine: from physiologic principles to health care application. Champaign (IL): Human Kinetics, 2004.

26. Rendell M, Bergman T, O’Donnell G, Drobny E, Borgos J, Bonner RF. Microvascular blood flow, volume and velocity measured by laser Doppler techniques in IDDM. Diabetes. 1999;38:819-24. 